\title{
Assess the Age of Majority Using Lower Wisdom Teeth
}

\section{Brkic $H^{*}$ \\ Department of Dental Anthropology, University of Zagreb School of Dental Medicine, Croatia}

*Corresponding author: Hrvoje Brkic, President of IOFOS, Chair of Forensic Dentistry, Department of Dental Anthropology, University of Zagreb School of Dental Medicine, Croatia; Email: brkic@sfzg.hr

\section{Mini Review}

This article is signed by 27 authors from four continents led by Roberto Cameriere one of the most cited authors in the field of dental age assessment. Determining dental age is a very important segment in the forensic sciences because it gives the best results for an individual's age. This is especially important on found dead bodies, human remains, but also on living people because by determining the dental age one can estimate the age of majority after 18 years of age. A large number of refugees who cross borders in search of asylum usually come without documents and passports. It is therefore difficult to determine the age of the majority of asylum seekers. The teeth are an important organ for determining dental age both in young people and in adults and the elderly. This paper describes a method of determining the dental age on the left and right lower wisdom teeth of a total of 10,181 orthopantomograms from Europe, Africa, Asia and America.

The sample selection criteria were as follows: clear OPGs and all permanent teeth present, including left and right third molars. The presence of any visible dental and bone pathology (e.g. large carious lesions or endodontic treatments), children with any systemic diseases or endocrine anomalies (e.g. dysmorphology, abnormally short roots) and subjects with previous root canal treatment in the lower permanent teeth were excluded from the study. Heavily rotated and impacted third molar with no visible roots were also excluded from this study. In 2021, Angelakopoulos N, et al. [1] developed a new dental method for assessing adult age. This method is based on the relationship between age and the normalized measures of the third molar's open apices, known as the third molar maturity index (I3M). All the statistical analyzes (Kolmogorov - Smirnov test, Wilcoxon Signed Rank test and Specifc descriptive statistics) were performed using the $\mathrm{R}$ software (version 3.6.3, R Core Team, R Foundation for
Statistical Computing, Vienna, Austria) and SPSS software (version 26.0, Armonk, IBM Corporation, NY, USA), adopting a signifcance level of $5 \%$.

\section{Conclusion}

This is the first work in which I3M is explored on both left and right lower third molar, utilizing a vast sample with origins in four different continents: Africa, America, Asia and Europe. The results presented here validated the use of both left and right lower wisdom teeth in the process of assessing age in cases of legal inquiry on the 18-year-old threshold since the accuracy attained for I3M on both right and left sides was high and did not difer signifcantly. This work indicates that there is a need to further explore data interpretation in their transition to legal and civil contexts. Concerning the current challenges and future perspectives, this research could be the foundation for a further novel study in which a combined approach left - right third molar is reproduced on a potentially larger dataset and by applying an appropriate statistical methodology to verify its usefulness in obtaining better age assessment. Further studies can include and analyze all four third molars and compare the results with cases that consider one or both mandibular third molars.

\section{Reference}

1. Angelakopoulos N, Galić I, Balla SB, Kiş HC, Jiménez LG, et al. (2021) Comparison of the third molar maturity index (I3M) between left and right lower third molars to assess the age of majority: a multi-ethnic study sample. Int J Legal Med. 